\title{
Epigenomic analysis of lung adenocarcinoma reveals novel DNA methylation patterns associated with smoking
}

This article was published in the following Dove Press journal:

OncoTargets and Therapy

18 October 2013

Number of times this article has been viewed

\author{
Qiang Tan',* \\ Guan Wangl,* \\ Jia Huang' \\ Zhengping Ding' \\ Qingquan Luo' \\ Tony Mok ${ }^{2}$ \\ Qian Tao ${ }^{2}$ \\ Shun Lu' \\ 'Department of Shanghai Lung Cancer \\ Center, Shanghai Chest Hospital, \\ School of Medicine, Shanghai Jiao Tong \\ University, Shanghai, People's Republic \\ of China; ${ }^{2}$ Cancer Epigenetics \\ Laboratory, Department of Clinical \\ Oncology, State Key Laboratory of \\ Oncology in South China, Sir YK Pao \\ Center for Cancer and Li Ka Shing \\ Institute of Health Sciences, The \\ Chinese University \\ of Hong Kong, Hong Kong \\ *These authors contributed equally to \\ this paper
}

\begin{abstract}
The importance of epigenetic regulation has been increasingly recognized in the development of cancer. In this study, we investigated the impact of smoking, a major risk factor of lung cancer, on DNA methylation by comparing the genome-wide DNA methylation patterns between lung adenocarcinoma samples from six smokers and six nonsmokers. We identified that smoking-induced DNA methylations were enriched in the calcium signaling and neuroactive ligand receptor signaling pathways, which are closely related to smoking-induced lung cancers. Interestingly, we discovered that two genes in the mitogen-activated protein kinase signaling pathway (RPS6KA3 and ARAF) were hypomethylated in smokers but not in nonsmokers. In addition, we found that the smoking-induced lung cancer-specific DNA methylations were mostly enriched in nuclear activities, including regulation of gene expression and chromatin remodeling. Moreover, the smoking-induced hypermethylation could only be seen in lung adenocarcinoma tissue but not in adjacent normal lung tissue. We also used differentially methylated DNA loci to construct a diagnostic model to distinguish smoking-associated lung cancer from nonsmoking lung cancer with a sensitivity of $88.9 \%$ and specificity of $83.2 \%$. Our results provided novel evidence to support that smoking can cause dramatic changes in the DNA methylation landscape of lung cancer, suggesting that epigenetic regulation of specific oncogenic signaling pathways plays an important role in the development of lung cancer.
\end{abstract}

Keywords: lung cancer, epigenome, methylation, tumor suppressor gene, smoking

\section{Introduction}

Lung cancer is the leading cause of cancer-related death worldwide. The estimated 5 -year survival rate ranges from $6 \%$ to $16 \%$, depending on the subtype. ${ }^{1}$ The tumorigenesis of lung cancer not only involves various genetic alterations, such as point mutations, deletions, and gene amplifications, but also alterations in epigenetics, including DNA methylation and histone modifications. One of the most studied epigenetic regulations is cytosine methylation, which does not affect the primary structure of chromatin, but does affect the secondary structure, which is critical to regulation of gene expression.

In lung cancer, upregulation of DNA methyltransferase 1 (DNMT1) protein has been shown to correlate with increased hypermethylation of tumor suppressor genes and downregulation of tumor suppressor proteins. ${ }^{2-4}$ Recently, Belinsky evaluated the methylation of genes isolated from blood samples in a cohort of women with different levels of risk for lung cancer. ${ }^{5}$ They identified that p16Ink4, MGMT, and RASSF1A were hypermethylated in the high-risk cohort. The methylation pattern of specific genes may be useful in early detection of lung cancer. In another study, Feng et al studied
Correspondence: Shun Lu or Qian Tao Shanghai Lung Tumor Clinical Medical Center, Shanghai Chest Hospital, Shanghai Jiao Tong University, Shanghai 200030, People's Republic of China

Tel 862162821990

Fax 862162804970

Email shun_lu@hotmail.com or qtao@ clo.cuhk.edu.hk 
the DNA methylation status of 27 genes from 49 patients with non-small cell lung cancer and found that some cancerspecific changes in DNA methylation pattern can only be seen in tumor tissues but not in preneoplastic tissues. ${ }^{6}$

Smoking, as a well known risk factor for lung cancer, has been widely recognized as a carcinogen with a significant role in tumorigenesis of the lung. Many studies have demonstrated that smoking can induce genomic instability by producing genetic mutations and altering epigenetic modifications. Hypermethylation of promoter regions have frequently been observed in smokers with and without lung cancer, which is consistent with the higher level of DNMT1 in lung samples from smokers when compared with that from nonsmokers. ${ }^{3,7,8}$ The cumulative smoking dose (pack-years) has been shown to correlate well with the frequency of methylation in cancerfree heavy smokers. ${ }^{9} C C N D 2$ and $A P C$ were frequently hypermethylated in both cancerous and noncancerous lung tissues of smokers with non-small cell lung cancer, suggesting that hypermethylation of these genes may be associated with environmental factors, such as chronic smoking. Other recent research showed that methylation of $R A S S F 1 A$ is associated with exposure to smoke in lung cancer. ${ }^{10,11}$ Hypermethylation of FHIT has been shown to be an early event in smoking-associated squamous cell lung cancer. ${ }^{12}$

On the other side, some studies have shown that smoking is not clearly associated with an altered DNA methylation pattern in lung cancer patients. Tommasi et al investigated the effects of chronic exposure to a prototype smoke-derived carcinogen, benzo[a]pyrene diol epoxide (B[a]PDE), on DNA methylation in genomic regions that have been previously demonstrated to be important in lung cancer. ${ }^{13}$ They demonstrated that chronic treatment of normal human cells in vitro with $\mathrm{B}[\mathrm{a}] \mathrm{PDE}$ did not alter the DNA methylation pattern in the genomic regions relevant to lung cancer within a time frame that preceded cellular transformation. ${ }^{13}$ Another study by Scesnaite et al did not identify significant changes in the pattern of DNA methylation associated with smoking. ${ }^{14}$ Hillemacher et al investigated the effect of smoking on the global DNA methylation pattern in 298 genomic DNA samples, but did not find a direct effect of smoking on global DNA methylation. ${ }^{15}$ However, these epigenetic studies were mostly focused on special gene groups or genome loci rather than systematic epigenome-wide analysis of DNA methylation pattern. Moreover, these studies relied on a qualitative methylation-specific polymerase chain reaction method that is subjective, relying on detection of a band from electrophoresis, which does not distinguish low-level from high-level methylation. ${ }^{16-18}$
In this study, we used an epigenome-wide screening method based on Illumina 27K DNA methylation microarray technology to study how smoking contributes to the development of lung cancer from a DNA methylation point of view. We identified differentially methylated genes by comparing the global DNA methylation patterns between lung adenocarcinoma samples from smokers and nonsmokers. Our study provides an insightful perspective on smoking-associated DNA methylation and its role in tumorigenesis of the lung.

\section{Materials and methods Sample preparations}

We obtained lung adenocarcinoma cancer tissues and corresponding adjacent normal lung tissues from 12 patients diagnosed with stage I-IIIa lung adenocarcinoma (six smokers and six nonsmokers) at the Shanghai Chest Hospital. All 12 patients enrolled in this study were untreated before they underwent complete tumor resection at the Shanghai Chest Hospital in 2010. Tumor staging was based on biopsy and conducted by pathologists in the Shanghai Chest Hospital. Baseline characteristics and tumor stages for all patients are listed in Supplementary Table 1. This study was approved by the ethics committee of the Shanghai Chest Hospital and the School of Medicine, Shanghai Jiao Tong University. All patients provided their written informed consent.

\section{Illumina 27K DNA methylation microarray}

The experiment was performed according to Illumina's protocol. Briefly, the procedures included:

1. Bisulfite treatment, whereby $1 \mu \mathrm{g}$ of genomic DNA was used in bisulfite conversion to convert the unmethylated cytosine into uracil.

2. Genomic DNA amplification, where the bisulfite-treated DNA was subjected to whole genome analysis by random hexamer primer and Phi29 DNA polymerase. The products were then enzymatically fragmented, purified from dNTPs, primers, and enzymes, before being applied to microarray chips.

3. Hybridization and single-base extension. There were two bead types for each $\mathrm{CpG}$ site per locus in the chip, which were differentiated by different bead types. A total of 200,000 beads were available in the chip. Hybridization was followed by single-base extension with haptenlabeled dideoxynucleotides.

4. Fluorescence staining and chip scanning. Multilayered immunohistochemical assays were performed by repeated rounds of staining with a combination of antibodies to 
differentiate the two types. After staining, the chip was scanned to show the intensities of the unmethylated and methylated bead types. The fluorescence intensity ratios between the two bead types were then calculated.

5. Analysis of methylation data. The scanned microarray images were analyzed using BeadStudio software (Illumina, San Diego, USA), which performed statistical tests on the results and normalized the raw data to reduce the effects of experimental variation and background.

\section{Analysis of DNA methylation data}

We used the MethyLumiSet Bioconductor package to handle the methylation microarray data as a data container in Methyllumi R. We studied the pattern of missing values and performed data imputation. Probes were discarded if they lay on the sex chromosomes. We then performed the Wilcoxon test to find significant differential DNA methylation regions between: normal lung tissues from smokers and normal lung tissues from nonsmokers to identify smokingspecific DNA methylation; and between lung cancer tissues from smokers and lung cancer tissues from nonsmokers to identify smoking-associated methylation changes that are specific to lung cancer.

\section{Gene ontology and pathway analysis}

We used hypergeometric analysis to identify the differentially methylated region (DMR) and obtained the DMR gene-enriched functional pathways. Gene function annotation was performed using resources from Gene Ontology. Pathway resources were obtained from KEGG and BioCarta. Gene functional cluster analysis was performed using the DAVID system.

\section{Construction of a diagnostic model for classifying smoking-associated and nonsmoking lung cancer}

We used the proper signature selection method, which combines the nearest centroid and genetic algorithm fitting tuning approach, to search for the best DNA methylation loci as diagnostic markers. Genetic algorithms are variable search procedures that are based on the principle of evolution by natural selection. The procedure works by evolving sets of variables (chromosomes) that fit certain criteria from an initial random population via cycles of differential replication, recombination, and mutation of the fittest chromosomes. ${ }^{19}$ The concept of using in silico evolution for resolution of optimization problems was introduced by John Holland in $1975 .{ }^{19}$

\section{Results}

\section{Identification of smoking-specific DNA methylation in lung adenocarcinoma}

To investigate how smoking affects DNA methylation in lung adenocarcinoma, we used a DNA methylation microarray to compare the global DNA methylation pattern between lung adenocarcinoma samples from smokers and nonsmokers (six samples for each group) to identify smoking-induced DNA methylations that are specific to lung adenocarcinoma (Figure 1A). We identified 137 DMRs, including a number of genes having been previously shown to function in smoking and cancer, such as $A D R B 3, G A L R 1$, and TRPC1. Of the $137 \mathrm{DMRs}, 48$ were hypermethylated in smokers with lung adenocarcinoma compared with nonsmokers with lung adenocarcinoma, while 89 of the 137 DMRs were hypomethylated. To filter out the smoking-induced DNA methylations that are not specific to lung adenocarcinoma, we also compared the global DNA methylation pattern for adjacent normal lung tissues from the same six smokers and six nonsmokers. We discovered 90 DMRs that were specifically induced by smoking, including two pro-oncogenic genes from the Ras/ Raf/MEK/ERK (mitogen-activated protein kinase, MAPK) signaling pathway ( $R P S 6 K A 3$ and $A R A F$ ). Interestingly, all 90 DMRs were hypomethylated loci (Figure 1B and C). By comparing the two sets of results, we found that 27 DMRs (all hypomethylated) can be found in both sets of results, indicating that these $27 \mathrm{DMRs}$ are smoking-specific changes in DNA methylation that present in both lung cancer tissues and normal lung tissues of smokers. After subtracting the 27 common DMRs from the 137 DMRs that we identified in the comparison between lung cancer tissues from smokers and nonsmokers, we obtained 110 smoking-induced DNA methylations specific to lung adenocarcinoma, including 48 hypomethylated DMRs and 62 hypermethylated DMRs (Figure 1B and C). The full list of the differentially methylated genes can be found in Supplementary Tables 2-4.

To gain a functional insight from our data, we performed a Gene Ontology analysis of all candidate genes. As a result, we found that the 90 smoking-induced DMRs in the normal lung tissues of smokers were enriched in pathways including energy metabolism, phosphorylase kinase activity, and ubiquitin thiolesterase activity (Table 1). The 110 smokinginduced DMRs specific to lung cancer were mostly enriched in biological processes associated with transcription factor activity, chromatin remodeling, and neural signal processing (Table 2). Using the data we obtained from this study, we proposed a model to depict the developmental progress 


\begin{tabular}{|l|c|c|}
\hline & Smoker tumor tissue STT & Smoker lung tissue SLT \\
\hline Nonsmoker tumor tissue NSTT & + & \\
\hline Nonsmoker lung tissue NSLT & & + \\
\hline
\end{tabular}

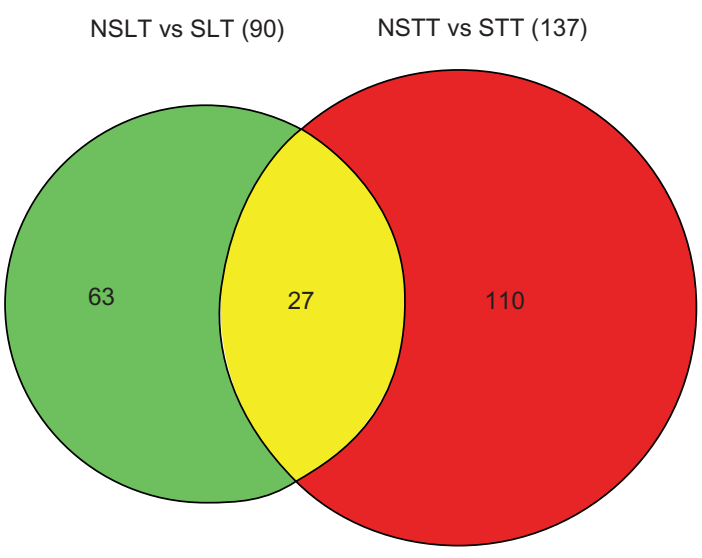

- Hypomethylation -Hypermethylation
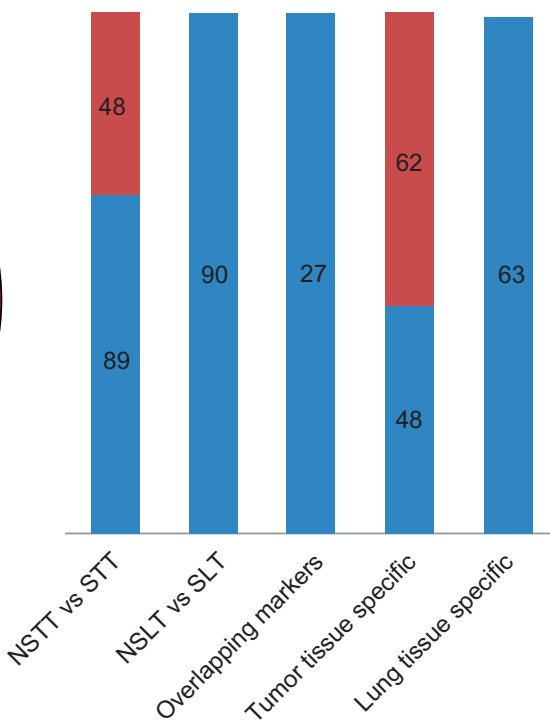

Figure I Differences in global DNA methylation pattern in lung cancer between smokers and nonsmokers. The global DNA methylation pattern in normal lung tissues from smokers (SLT) was compared with that from nonsmokers (NSLT), producing 90 differentially methylated regions. The global DNA methylation pattern of lung tumor tissues from smokers (STT) was compared with that from nonsmokers (NSTT), resulting in 137 differentially methylated regions. Twenty-seven loci can be found in both result sets. The numbers of hypermethylation and hypomethylation loci are also shown.

Abbreviation: vs, versus.

of smoking-induced lung cancer from an epigenetic point of view (Figure 2).

\section{Diagnosis model for distinguishing smoking-associated from nonsmoking lung cancer}

To translate our results into clinical practice, we aimed to use the smoking-specific DNA methylation pattern as a diagnosis tool to classify lung cancer samples associated with smoking and those that are not. We thus used a machine learning algorithm known as the nearest centroid approach, which is a combined model using a tuning strategy of genetic algorithms to select robust DNA methylation markers to classify smoking and nonsmoking lung cancers. We found that a set of differentially methylated DNA methylation loci can predict smoking-associated lung cancer and nonsmoking lung cancer with a sensitivity of $83.2 \%$ and a specificity of $88.9 \%$. The overall accuracy and principal component analysis (PCA) of the classification model is shown in Figure 3.

\section{Discussion}

Epigenetic regulation has emerged to become an important field in studying the development of cancer. In this study, we conducted an epigenome-wide screening of DNA

Table I Pathways enriched with differentially methylated genes from the comparison between normal lung tissues from smokers and nonsmokers

\begin{tabular}{|c|c|c|c|c|}
\hline Category & Term & Count & $\%$ & $P$ \\
\hline GOTERM_BP_FAT & GO:0006006 glucose metabolic process & 4 & 7.843137255 & 0.011044244 \\
\hline GOTERM_MF_FAT & GO:0004689 phosphorylase kinase activity & 2 & 3.921568627 & 0.014170587 \\
\hline GOTERM_MF_FAT & GO:000422I ubiquitin thiolesterase activity & 3 & $5.88235294 \mid$ & 0.019246991 \\
\hline GOTERM_BP_FAT & GO:0019318 hexose metabolic process & 4 & 7.843137255 & 0.0202083 \\
\hline GOTERM_BP_FAT & GO:0005996 monosaccharide metabolic process & 4 & 7.843137255 & 0.029419949 \\
\hline GOTERM_MF_FAT & GO:0016790 thiolester hydrolase activity & 3 & $5.88235294 \mid$ & 0.034057248 \\
\hline GOTERM_MF_FAT & GO:0000 I66 nucleotide binding & 12 & $23.5294 I I 76$ & 0.04365391 \\
\hline GOTERM_MF_FAT & GO:0048487 beta-tubulin binding & 2 & 3.921568627 & $0.04466|57|$ \\
\hline
\end{tabular}


Table 2 Pathway enrichment analysis for smoking-induced lung cancer-specific differentially methylated genes

\begin{tabular}{|c|c|c|c|}
\hline Term & Count & $\%$ & $\mathbf{P}$ \\
\hline GO:0003700 transcription factor activity & 10 & 27.77777778 & 0.000326 \\
\hline GO:0019932 second messenger-mediated signaling & 5 & I3.88888889 & 0.001931974 \\
\hline GO:0043565 sequence-specific DNA binding & 7 & 19.44444444 & 0.00275705 \\
\hline GO:0048I68 regulation of neuronal synaptic plasticity & 3 & 8.333333333 & 0.003050078 \\
\hline GO:0050804 regulation of synaptic transmission & 4 & $\mid I . I I I I I I I$ & 0.003636035 \\
\hline GO:005 I969 regulation of transmission of nerve impulse & 4 & II.IIIIIIII & 0.004521842 \\
\hline GO:003 I 644 regulation of neurologic system process & 4 & II.IIIIIIII & 0.005055558 \\
\hline GO:0003677 DNA binding & 13 & 36.111111111 & 0.005249171 \\
\hline GO:0030528 -transcription regulator activity & 10 & 27.77777778 & $0.007 \mid 37687$ \\
\hline GO:0048I67 regulation of synaptic plasticity & 3 & 8.333333333 & 0.009379318 \\
\hline GO:0003002 regionalization & 4 & II.IIIIIIII & 0.010129184 \\
\hline GO:0006325 chromatin organization & 5 & | 3.88888889 & 0.010398849 \\
\hline GO:0006334 nucleosome assembly & 3 & 8.333333333 & 0.015765103 \\
\hline GO:003 I497 chromatin assembly & 3 & 8.333333333 & 0.016846638 \\
\hline GO:0030I82 neuron differentiation & 5 & | 3.88888889 & 0.017077758 \\
\hline GO:00480I5 phosphoinositide-mediated signaling & 3 & 8.333333333 & 0.017214047 \\
\hline GO:0065004 protein-DNA complex assembly & 3 & 8.333333333 & 0.018336737 \\
\hline GO:0034728 nucleosome organization & 3 & 8.333333333 & 0.019102083 \\
\hline GO:0007389 pattern specification process & 4 & $\mid I .11 I I I I I$ & 0.022739147 \\
\hline GO:005 I 276 chromosome organization & 5 & |3.88888889 & 0.023859659 \\
\hline GO:0006323 DNA packaging & 3 & 8.333333333 & 0.029291461 \\
\hline GO:0044057 regulation of system process & 4 & $\mid 1.11111111$ & 0.033103168 \\
\hline GO:0006333 chromatin assembly or disassembly & 3 & 8.333333333 & 0.03405299 \\
\hline GO:0034622 cellular macromolecular complex assembly & 4 & $\mid I .11111111$ & 0.035597851 \\
\hline GO:0048666 neuron development & 4 & II.IIIIIIIII & 0.041790392 \\
\hline GO:0006355 regulation of transcription, DNA-dependent & 9 & 25.00000000 & 0.042198386 \\
\hline GO:0044093 positive regulation of molecular function & 5 & 13.88888889 & 0.043420144 \\
\hline GO:005 I 252 regulation of RNA metabolic process & 9 & 25.00000000 & 0.047283874 \\
\hline GO:003462 I cellular macromolecular complex subunit organization & 4 & II.IIIIIIII & 0.04750734 \\
\hline
\end{tabular}

methylation patterns in lung adenocarcinoma samples from smokers and nonsmokers. By comparing normal lung tissue samples between smokers and nonsmokers, we identified 90 differentially methylated regions comprising genes functioning in the energy metabolism process and mitogenic kinase activity, suggesting that smoking alters cellular energy supply and cell cycle regulation in normal lung cells. Since both aberrant energy metabolism and a dysregulated cell cycle are the hallmarks of cancer, smoking makes lung cells prone to the development of neoplastic disease. Interestingly, all 90 DMRs were hypomethylated in normal lung tissues from smokers compared with those from nonsmokers. Global DNA hypomethylation has been well demonstrated in many types of cancers. ${ }^{20}$ However, unlike the close link between DNA hypermethylation and suppression of tumor suppressors, the role of DNA hypomethylation in the development of cancer is still elusive. ${ }^{21}$ Several lines of evidence support that DNA hypomethylation contributes to tumorigenesis

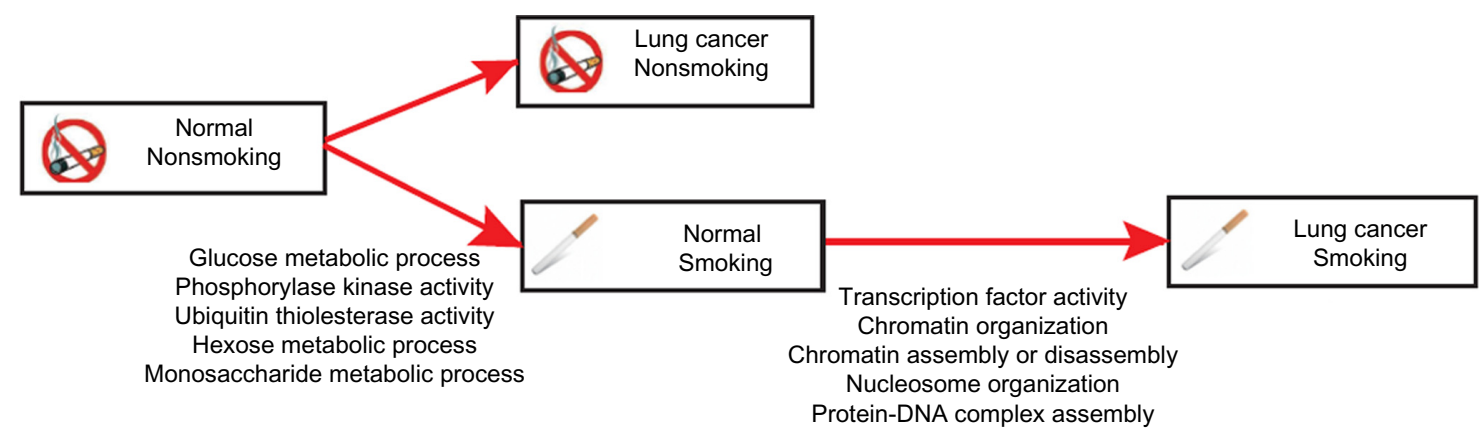

Figure 2 Hypothesized model of smoking-induced lung cancer based on our data. 

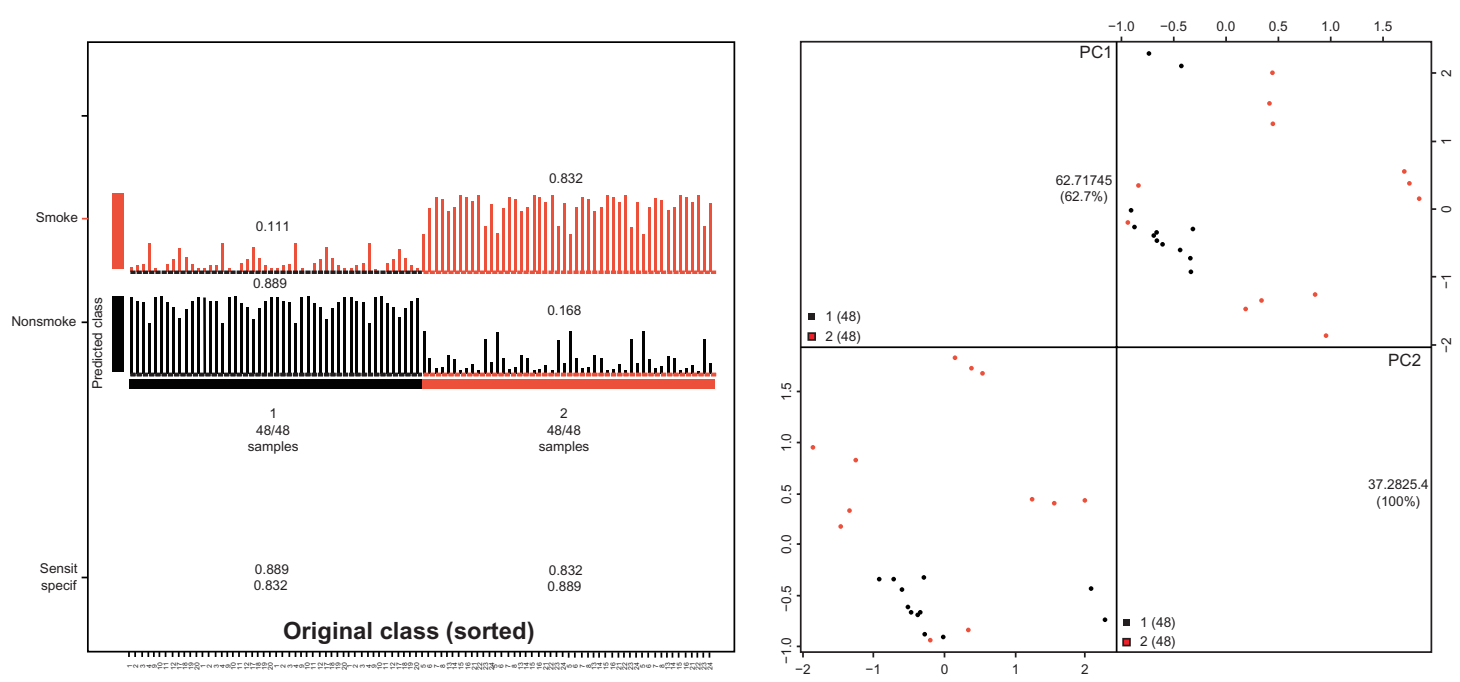

Figure 3 Diagnostic model for classifying smoking-associated lung cancer and nonsmoking lung cancer.

Notes: Classification accuracy is shown with the horizontal axis represents the individual samples grouped according to the disease class whereas the vertical axis represents the predicted classes. Black denotes nonsmokers and red denotes smokers.

Abbreviations: PC, principal component; Sensit, sensitivity; specif, specificity.

by inducing genomic instability. ${ }^{21}$ Our results indicate that smoking preferentially induces hypomethylation in normal lung tissues, which may predispose smokers to the development of lung cancer by inducing genomic instability.

By comparing smoking-specific DNA methylation with lung cancer-specific DNA methylation, we discovered 110 differentially methylated genes that were specifically associated with smoking-induced lung cancer. These genes are enriched in genetic and epigenetic regulatory pathways such as transcription factor activity and chromatin remodeling activity. This result indicates that smoking-induced tumorigenesis requires extensive nuclear reorganization and reflects the fundamental characteristic differences between smoking-induced lung cancer and lung cancer not related to smoking, and also provides a potential diagnostic method to distinguish smoking-induced lung cancer from lung cancer not related to smoking.

Many interesting genes that may function in smoking and cancer were identified in this study. We found that both RPS6KA3 and ARAF genes are hypomethylated in smokers but not in nonsmokers. Both genes take part in the MAPK kinase signaling pathway. The Ras/Raf/MEK/ERK (MAPK) signal transduction cascade is an important regulator of cell proliferation. RAS gene products activate proteins in the RAF family, which consists of ARAF, BRAF and RAF-1. In a lung cancer study, Lee et al identified the mutation spectrum revealed by paired genome sequences from a lung cancer patient, and suggested a model for how the multiplicity of mutations within the MAPK signaling cascade acted together to drive constitutive progrowth signals. ${ }^{22}$ They found that MAPK signaling genes ( $S H C 1, G R B 2, S O S, A R A F, A P 3 K 3$, and $E L K 1$ ) were upregulated in lung cancer. ${ }^{22,23}$ In addition, hypomethylation of MAPK signaling members in smokers is an indicator of oncogenic changes that may be valuable targets for early cancer detection. ${ }^{22,23}$

$A D R B 3$, a beta-adrenergic receptor, was hypermethylated in smokers with lung cancer but not in nonsmokers with lung cancer in this study. Recent studies have revealed that nitrosamine 4-(methylnitrosamino)-1-(3-pyridyl)-1-butanone (NNK) can directly bind and stimulate the beta-adrenergic receptor signaling pathway. NNK is derived from nicotine, and has been demonstrated to cause mutations in genes that affect cell regulation and proliferation. $A D R B 3$ also functions in activation of the neuroactive ligand receptor interaction pathway. In an earlier study, the researchers identified other common genetic variants associated with the risk of lung cancer in the NNK disposition pathways, such as CYP2A13, the most active $\mathrm{P} 450$ for phase metabolic activation of NNK and the receptor (ADRB2) in its nongenotoxic pathway. ${ }^{24}$ Masi et al verified the mutagenic effects of NNK in an animal model and provided a way of identifying mutations not only in $A D R B 2$ but also in other genes that may play an essential role in the development of lung adenocarcinoma. ${ }^{25}$ Therefore, it will be interesting to know if $A D R B 3$ also has a role in smoking-induced lung cancer.

GALR1 is another interesting gene we identified in this study that is specific to smoking-associated lung cancer. GALR1 is one of the three G protein-coupled receptors that 
function as the receptor for galanin, which is a multifunctional neuropeptide widely expressed in the mammalian central and peripheral nervous systems. Expression of galanin and its three receptors (GALR1-3) are silenced in several types of tumor. ${ }^{26}$ Hypermethylation of $G A L R 1$ has been correlated with reduced gene expression. Reported by Misawa et al, GALRl is silenced in head and neck squamous cell carcinoma because of its hypermethylated promoter. The methylation status of the GALR1 promoter and the level of GALR1 gene expression have been well correlated in a large panel of head and neck squamous cancer cell lines and primary tumor specimens. ${ }^{26}$ Ectopic expression of GALR1 suppresses tumor cell proliferation through Erk1/2-mediated regulations on cyclin-dependent kinase inhibitors and cyclin D1. ${ }^{27}$ Interestingly, Jackson et al utilized a mouse model to show that galanin has a protective role against neuropeptide dependence, and these effects may be mediated by GALR1. In addition, several lines of evidence support that galanin acts through GALR1 to modulate the physiologic effects of nicotine. ${ }^{28}$ However, the role of GALR 1 in smoking-induced lung cancer is still unclear and deserves further investigation.

Smoking-associated lung cancer often demonstrates clinical and pathologic characteristics different from lung cancers that are not associated with smoking. Hence, it has clinical value for classifying smoking-associated lung cancer and nonsmoking lung cancer. In this study, we used smoking-specific DNA methylation loci to construct a diagnostic model to predict whether development of lung cancer is associated with smoking. In this study, our results were obtained from lung adenocarcinoma, which is the most common subtype of lung cancer. Because different types of lung cancer have distinct clinicopathologic characteristics, it should be noted that the value of our diagnostic model for other types of lung cancer is still unknown. In the next stage of study, we will need to validate this model further in a larger patient cohort and in other types of lung cancer.

In summary, we examined differences in global DNA methylation patterns between smoker and nonsmoker groups of lung cancer patients to identify differentially methylated genes associated with smoking-induced lung cancer. Our results provide novel evidence that smoking plays an important role in the development of lung cancer through DNA methylation.

\section{Acknowledgments}

This research is supported by the Chinese Ministry of Science and Technology and the Sino-Swiss International Collaboration project (2012DFG31320). This study is supported by the Chinese Ministry of Science and Technology international collaboration foundation, project number 2010DFA32580. The authors thank all the patients who participated in this study.

\section{Disclosure}

The authors report no conflicts of interest in this work.

\section{References}

1. Tsou JA, Hagen JA, Carpenter CL, Laird-Offringa IA. DNA methylation analysis: a powerful new tool for lung cancer diagnosis. Oncogene. 2002;21:5450-5461.

2. Damiani LA, Yingling CM, Leng S, Romo PE, Nakamura J, Belinsky SA Carcinogen-induced gene promoter hypermethylation is mediated by DNMT1 and causal for transformation of immortalized bronchial epithelial cells. Cancer Res. 2008;68:9005-9014.

3. Lin RK, Hsieh YS, Lin P, et al. The tobacco-specific carcinogen NNK induces DNA methyltransferase 1 accumulation and tumor suppressor gene hypermethylation in mice and lung cancer patients. $J$ Clin Invest. 2010;120:521-532.

4. Liu F, Killian JK, Yang M, et al. Epigenomic alterations and gene expression profiles in respiratory epithelia exposed to cigarette smoke condensate. Oncogene. 2010;29:3650-3664.

5. Belinsky SA. Gene-promoter hypermethylation as a biomarker in lung cancer. Nat Rev Cancer. 2004;4:707-717.

6. Feng Q, Hawes SE, Stern JE, et al. DNA methylation in tumor and matched normal tissues from non-small cell lung cancer patients. Cancer Epidemiol Biomarkers Prev. 2008;17:645-654.

7. Baryshnikova E, Destro A, Infante MV, et al. Molecular alterations in spontaneous sputum of cancer-free heavy smokers: results from a large screening program. Clin Cancer Res. 2008;14:1913-1919.

8. Monick MM, Beach SR, Plume J, et al. Coordinated changes in AHRR methylation in lymphoblasts and pulmonary macrophages from smokers. Am J Med Genet B Neuropsychiatr Genet. 2008;159:141-151.

9. Ostrow KL, Hoque MO, Loyo M, et al. Molecular analysis of plasma DNA for the early detection of lung cancer by quantitative methylationspecific PCR. Clin Cancer Res. 2010;16:3463-3472.

10. Toyooka S, Maruyama R, Toyooka KO, et al. Smoke exposure, histologic type and geography-related differences in the methylation profiles of non-small cell lung cancer. Int J Cancer. 2003;103:153-160.

11. Kim DH, Ji YI. Hypermethylation of RASSF1A promoter is associated with the age at starting smoking and a poor prognosis in primary non-small cell lung cancer. Cancer Res. 2003;63:3743-3746.

12. Li W, Deng J, Jiang P, Tang J. Association of 5'-CpG island hypermethylation of the FHIT gene with lung cancer in southern-central Chinese population. Cancer Biol Ther. 2010;10:997-1000.

13. Tommasi S, Kim S, Zhong XY, Wu XW, Pfeifer GP, Besaratinia A. Investigating the epigenetic effects of a prototype smoke-derived carcinogen in human cells. PLoS One. 2010;5:e10594.

14. Scesnaite A, Jarmalaite S, Mutanen P, et al. Similar DNA methylation pattern in lung tumours from smokers and never-smokers with secondhand tobacco smoke exposure. Mutagenesis. 2011;27:423-429.

15. Hillemacher T, Frieling H, Moskau S, Muschler MA, Semmler A, Kornhuber J. Global DNA methylation is influenced by smoking behaviour. Eur Neuropsychopharmacol. 2008;18:295-298.

16. Shivapurkar SV, Suzuki M. Application of a methylation gene panel by quantitative PCR for lung cancers. Cancer Lett. 2007;247:56-71.

17. Vallbohmer BJ, Yang D. DNA methyltransferases messenger RNA expression and aberrant methylation of $\mathrm{CpG}$ islands in non-small-cell lung cancer: association and prognostic value. Clin Lung Cancer. 2006;8:39-44.

18. Kim YT, Sung SW, Kim JH. Can aberrant promoter hypermethylation of $\mathrm{CpG}$ islands predict the clinical outcome of non-small cell lung cancer after curative resection? Ann Thorac Surg. 2005;79:1180-1188. 
19. Levner I. Feature selection and nearest centroid classification for protein mass spectrometry. BMC Bioinformatics. 2005;6:68.

20. Jones PA. Functions of DNA methylation: islands, start sites, gene bodies and beyond. Nat Rev Genet. 2012;13:484-492.

21. De Smet C, Loriot A. DNA hypomethylation in cancer: epigenetic scars of a neoplastic journey. Epigenetics. 2010;5:206-213.

22. Lee W, Jiang ZS, Liu JF, et al. The mutation spectrum revealed by paired genome sequences from a lung cancer patient. Nature. 2010;465: 473-477.

23. Dhillon AS, Hagan S, Rath O, Kolch W. MAP kinase signalling pathways in cancer. Oncogene. 2007;26:3279-3290.

24. Al-Wadei HA, Schuller HM. Nicotinic receptor-associated modulation of stimulatory and inhibitory neurotransmitters in NNK-induced adenocarcinoma of the lungs and pancreas. J Pathol. 2008;218: $437-445$.
25. Masi T, Cekanova M, Walker K, et al. Nitrosamine 4-(methylnitrosamino)1-(3-pyridyl)-1-butanone-induced pulmonary adenocarcinomas in Syrian golden hamsters contain beta 2 -adrenergic receptor singlenucleotide polymorphisms. Genes Chromosomes Cancer. 2005;44: 212-217.

26. Misawa K, Ueda Y, Kanazawa T, et al. Epigenetic inactivation of galanin receptor 1 in head and neck cancer. Clin Cancer Res. 2008;14: 7604-7613.

27. Kanazawa T, Kommareddi PK, Iwashita T, et al. Galanin receptor subtype 2 suppresses cell proliferation and induces apoptosis in $\mathrm{p} 53$ mutant head and neck cancer cells. Clin Cancer Res. 2009;15:2222-2230.

28. Jackson KJ, Chen X, Miles MF, Harenza J. The neuropeptide galanin and variants in the GalR1 gene are associated with nicotine dependence. Neuropsychopharmacology. 2011;36:2339-2348. 


\section{Supplementary tables}

Supplementary tables 1-4 http://www.dovepress.com/cr data/supplementary file 51041.pdf.

\section{Publish your work in this journal}

OncoTargets and Therapy is an international, peer-reviewed, open access journal focusing on the pathological basis of all cancers, potential targets for therapy and treatment protocols employed to improve the management of cancer patients. The journal also focuses on the impact of management programs and new therapeutic agents and protocols on
Dovepress

patient perspectives such as quality of life, adherence and satisfaction. The manuscript management system is completely online and includes a very quick and fair peer-review system, which is all easy to use. Visit http://www.dovepress.com/testimonials.php to read real quotes from published authors.

\footnotetext{
Submit your manuscript here: http://www.dovepress.com/oncotargets-and-therapy-journal
} 